H. Tsuji

Nagoya Math. J.

Vol. 142 (1996), 5-16

\title{
GLOBAL GENERATION OF ADJOINT BUNDLES
}

\author{
HAJIME TSUJI
}

\section{Introduction}

In 1988, I. Reider proved that for a smooth projective surface $X$ and an ample line bundle $L$ on $X, K_{X}+3 L$ is globally generated and $K_{X}+4 L$ is very ample ([12]). In fact his theorem is much stronger than this (see [12] for detail). Recently a lot of results have been obtained about effective base point freeness (cf. $[1,3,8,13,14,15])$. In particular J. P. Demailly proved that $2 K_{X}+12 n^{n} L$ is very ample for a smooth projective $n$-fold $X$ and an ample line bundle $L$ on $X$. [2] will give a good overview for these recent results. The motivation of these works is the following conjecture posed by T. Fujita.

Conjecture ([4]). Let $X$ be a smooth projective $n$-fold defined over $\mathbf{C}$ and let $L$ be an ample line bundle on $X$. Then $K_{X}+(n+1) L$ is generated by global sections and $K_{X}+(n+2) L$ is very ample.

We note that Fujita's conjecture is trivial if $L$ is very ample by induction on $\operatorname{dim} X$. In the above situation, it is easy to see that $K_{X}+(n+1) L$ is nef and $K_{X}+(n+2) L$ is ample by using the theory of extremal rays (Mori theory cf. $([10,6])$. Moreover by using the base point free theorem ([7, p. 581, Theorem 6.1]), $K_{X}+(n+1) L$ is semiample, i.e. there exists a positive integer $m$ such that $m\left(K_{X}\right.$ $+(n+1) L)$ is generated by global sections. The number $n+1$ is nothing but the maximal length of extremal rays of smooth projective $n$-folds. In this paper, we shall prove the following theorem.

Theorem 1. Let $X$ be a smooth projective variety over $\mathbf{C}$ of dimension $n$ and let $L$ be an ample line bundle on $X$. Then $K_{X}+m L$ is generated by global sections on $X$ for every

$$
m \geq n(n+1) / 2+1 \text {. }
$$

Received December 19, 1994. 
The following two corollaries are the immediate consequence of Theorem 1 .

Corollary 1. Let $X$ be a smooth projective variety of dimension $n$ defined over $\mathbf{C}$ such that the canonical bundle $K_{X}$ is ample. Then $m K_{X}$ is generated by global sections for every $m \geq n(n+1) / 2+2$.

Corollary 2. Let $X$ be a smooth projective variety of dimension $n$ defined over $\mathbf{C}$ such that the anticanonical bundle $-K_{X}$ is ample. Then $-m K_{X}$ is generated by global sections for every $m \geq n(n+1) / 2$.

Our method is extremely simple. We hope this method is applicable to obtain effective bound for very ampleness of adjoint bundles.

The author would like to express his hearty thanks to Professor T. Ohsawa who pointed out an error in the first draft of this paper.

\section{Proof of Theorem 1}

In the proof of Theorem 1 we shall use singular hermitian metrics as in [1]. But our proof is mainly algebraic. For example we do not use Monge-Ampère equation.

\subsection{Singular hermitian metrics and a vanishing theorem}

Definition 1. Let $L$ be a line bundle over a complex manifold $M$. A singular hermitian metric $h$ on $L$ is given by

$$
h=e^{-\varphi} h_{0},
$$

where $h_{0}$ is a $C^{\infty}$-hermitian metric on $L$ an $\varphi \in L_{\text {loc }}^{1}(M)$ is an arbitrary function, called the weight of the metric with respect to $h_{0}$.

We define a closed current curv $h$ by

$$
\operatorname{curv} h=\operatorname{curv} h_{0}+\sqrt{-1} \partial \bar{\partial} \varphi,
$$

where curv $h_{0}$ is the curvature form of the hermitian metric $h_{0}$ and $\partial \bar{\partial}$ is taken in the sense of current. We call curv $h$ the curvature current of the singular hermitian line bundle $(L, h)$. It is easy to see that curv $h$ is independent of the choice of $h_{0}$ and $\varphi$. 
Definition 2. Let $T$ be a positive $(1,1)$ current on a complex manifold $M . T$ is said to be strictly positive, if for every point $x \in M$, there exists a neighbourhood $U$ of $x$ and $a C^{\infty}$ Kähler form $\omega$ on $U$ such that $T-\omega$ is a positive $(1,1)$-current on $U$.

Definition 3. Let $L$ be a line bundle on a complex manifold $M$ and let $h$ be a singular hermitian metric on $L$. The $L^{2}$-sheaf $\mathscr{L}^{2}(L, h)$ is the sheaf defined by

$$
\mathscr{L}^{2}(L, h)(U)=\left\{\sigma \in \Gamma(U, L) \mid h(\sigma, \sigma) \in L_{\mathrm{loc}}^{1}(U)\right\} .
$$

We shall recall the following theorem.

Theorem 2 ([11] (see also [1, p. 333, Theorem 4.5]). Let $X$ be a smooth projective variety and let $L$ be a line bundle on $X$. Let $h$ be a singular hermitian metric on $L$ such that curv $h$ is strictlty positive. Then $\mathscr{L}^{2}(L, h)$ is a coherent sheaf of $\mathscr{O}_{X}$ module and

$$
H^{p}\left(X, \mathfrak{O}_{X}\left(K_{X}\right) \otimes \mathscr{L}^{2}(L, h)\right)=0
$$

holds for every $p \geq 1$.

\subsection{Construction of singular hermitian metrics}

Let $X$ be a smooth projective variety of dimension $n$ defined over $\mathbf{C}$ and let $L$ be an ample line bundle on $X$. Let $x \in X$ be a point. We shall construct a singular hermitian metric on some multiple of $L$ with sufficiently large singularity at $x$ and (semi) positive curvature in the sense of current.

Lemma 1. For sufficiently large $H^{0}\left(X, \mathscr{O}_{X}(m(2 n+1) L) \otimes \mathcal{M}_{x}^{\otimes 2 m n}\right)$ is not zero, where $\mathcal{M}_{x}$ denotes the ideal sheaf of $x$.

Proof. Let us consider the exact sequence:

$$
\begin{aligned}
0 \rightarrow H^{0}\left(X, \mathscr{O}_{X}(m(2 n+1) L) \otimes \mathcal{M}_{x}^{\otimes 2 m n}\right) & \rightarrow \\
H^{0}\left(X, \mathcal{O}_{X}(m(2 n+1) L)\right) & \rightarrow \mathcal{O}_{X}(m(2 n+1) L) / \mathcal{M}_{x}^{\otimes 2 m n} .
\end{aligned}
$$

$\mathfrak{O}_{X} / M_{x}^{\otimes 2 m n}$ is a skyscraper sheaf of rank $\left(\begin{array}{c}2 m n+n-1 \\ n\end{array}\right)$. On the other hand by Serre's vanishing theorem and Riemann-Roch theorem, we see that 


$$
\operatorname{dim} H^{0}\left(X, \mathscr{O}_{X}((2 n+1) m L)\right)=\frac{(2 n+1)^{n} L^{n}}{n !} m^{n}+O\left(m^{n-1}\right)
$$

holds. Since

$$
\left(\begin{array}{c}
2 m n+n-1 \\
n
\end{array}\right)=\frac{2^{n} n^{n}}{n !} m^{n}+O\left(m^{n-1}\right)
$$

holds, we see that

$$
H^{0}\left(X, \mathscr{O}(m(2 n+1) L) \otimes \mathcal{M}_{x}^{\otimes 2 m n}\right) \neq 0
$$

holds for sufficiently large $m$.

Remark 1. There is no particular reason to use the number 2 in Lemma 1, i.e. for any fixed positive integer $N$, we can prove that

$$
H^{0}\left(X, \mathscr{O}_{X}(m(N n+1) L) \otimes \mathcal{M}_{x}^{\otimes N m n}\right) \neq 0
$$

for a sufficiently large $m$. This fact will be used later.

For simplicity we set

$$
\Lambda_{m}=\left|H^{0}\left(X, \mathscr{O}_{X}(m(2 n+1) L) \otimes \mathcal{M}_{x}^{\otimes 2 m n}\right)\right| .
$$

We consider $\Lambda_{m}$ as a linear subsystem of $|m(2 n+1) L|$. We set

$$
B_{m}=\operatorname{Bs} \Lambda_{m} \text {. }
$$

Let us take a $\mathbf{C}$ basis $\sigma_{0}, \ldots, \sigma_{N}$ of $H^{0}\left(X, \mathscr{O}_{X}(m(2 n+1) L) \otimes \mathcal{M}_{x}^{\otimes 2 m n}\right)$. Then $\sigma_{0}, \ldots, \sigma_{N}$ generates the ideal sheaf of the scheme $B_{m}$ over $\mathscr{O}_{X}$. Let $h$ be a $C^{\infty}$ hermitian metric of $L$ such that curv $h$ is a Kähler form on $X$. We define a singular hermitian metric $H_{x}$ of $\mathfrak{O}_{X}(2 m(n+1) L)$ by

$$
H_{x}=\frac{h^{\otimes m(2 n+1)}}{\sum_{k=0}^{N} h^{\otimes m(2 n+1)}\left(\sigma_{k}, \sigma_{k}\right)} .
$$

Let us define a closed current $T$ by

$$
T=\operatorname{curv} H_{x} .
$$

Let $\Phi_{x}: X-\cdots \rightarrow \mathbf{P}^{N}$ be the rational map defined by

$$
\Phi_{x}(p)=\left[\sigma_{0}(p): \ldots: \sigma_{N}(p)\right]
$$

Then $T$ is expressed by 


$$
T=\Phi^{*} \omega_{F S},
$$

where $\omega_{F S}$ denotes the Fubini-Study Kähler form of $\mathbf{P}^{N}$. Because $\Phi$ is only a rational map, we need to explain a little bit more the precise meaning of $\Phi^{*} \omega_{F S}$. Let $G \subset X \times \mathbf{P}^{N}$ be the graph of the rational map $\Phi$. Let $\pi_{i}(i=1,2)$ denote the restriction of the first and second projections on $G$ respectively. Then $\Phi^{*} \omega_{F S}$ is defined by

$$
\Phi^{*} \omega_{F S}=\left(\pi_{1}\right)_{*} \pi_{2}^{*} \omega_{F S} .
$$

This implies that $T$ is a closed positive current on $X$.

We shall analyze $T$.

\subsection{Basic invariant}

Let $H_{x}$ be the singular hermitian metric of $L^{\otimes m(2 n+1)}$ constructed in 2.2. Let us define a function on $\varphi$ on $X$ by

$$
\varphi=-\frac{1}{2 m} \log \left(\frac{H_{x}}{h^{\otimes m(2 n+1)}}\right) .
$$

For $t \in[0,1]$, we define an ideal sheaf $\mathscr{I}(t)$ by

$$
\mathscr{I}(t):=\mathscr{L}^{2}\left(\mathfrak{O}_{X}, e^{-t \varphi}\right) .
$$

If $s \leq t$, then

$$
\mathscr{I}(t) \subset \mathscr{I}(s)
$$

holds. By increasing $t$ from 0 to 1 , we obtain a strictly decreasing sequence of ideals:

$$
\mathfrak{O}_{X, x} \supset \mathscr{I}_{1, x} \supset \mathscr{I}_{2, x} \supset \cdots \supset \mathscr{I}_{k, x}
$$

We set

$$
\alpha=\sup \left\{t \in[0,1] \mid \mathscr{I}(t)_{x}=\mathscr{O}_{X, x}\right\}
$$

and

$$
V=\text { the union of irreducible components of } V \mathscr{I}_{1} \text { containing } x,
$$

where $V \mathscr{I}_{1}$ denotes the zero variety of $\mathscr{I}_{1}$. We note that $V$ is nonempty because $1 /\left(\sum_{i=1}^{n}\left|z_{i}\right|^{2}\right)^{n}$ is not locally integrable near the origin in $\mathbf{C}^{n}$. Then $V$ is a reduced (but may not be irreducible) subvariety of $X$. 


\subsection{Case: $\operatorname{codim} V=n$}

In this case $V=\{x\}$. Let us define a singular hermitian metric $h_{x}$ of $\mathscr{O}_{X}((n+$ 1) L) by

$$
h_{x}=H_{x}^{\frac{\alpha+\varepsilon}{2 m}} h^{\left(n+1-\left(n+\frac{1}{2}\right)(\alpha+\varepsilon)\right)},
$$

where $\varepsilon$ is a sufficiently small positive number. Then since

$$
\operatorname{curv} h_{x}=\frac{\alpha+\varepsilon}{2 m} T+\left(n+1-\left(n+\frac{1}{2}\right)(\alpha+\varepsilon)\right) \operatorname{curv} h,
$$

$h_{x}$ has strictly positive curvature. By Theorem 2 , we have

$$
H^{p}\left(X, \mathscr{O}_{X}\left(K_{X} \otimes \mathscr{L}^{2}\left(L^{\otimes(n+1)}, h_{x}\right)\right)\right)=0
$$

holds for every $p \geq 1$. We note that $x$ is an isolated point in the zero variety of $\Phi_{1}$. Hence

$$
H^{0}\left(X, \mathscr{O}_{X}\left(K_{X}+(n+1) L\right)\right) \rightarrow \mathscr{O}_{X}\left(K_{X}+(n+1) L\right) / \mathcal{M}_{x}
$$

is surjective. Hence $K_{X}+(n+1) L$ is generated by global sections at $x$.

\subsection{Case: codim $V<n$}

Let $X_{1}$ be a minimal dimensional irreducible component of $V$ and let $n_{1}$ be the dimension of $X_{1}$. For the first we assume that $X_{1}$ is nonsingular at $x$. The following lemma is an easy consequence of Serre's vanishing theorem.

LEMMA 2. The restriction morphism

$$
\phi: H^{0}\left(X, \mathscr{O}_{X}(\nu L)\right) \rightarrow H^{0}\left(X_{1}, \mathscr{O}_{X_{1}}\left(\nu L \mid X_{1}\right)\right)
$$

is surjective for every sufficiently large $\nu$.

Lemma 3. Let $x \in X_{1}$ be a regular point of $X_{1}$, then

$$
\left.H^{0}\left(X_{1}, \mathcal{O}_{X_{1}}(2 n+1) m_{1} L \mid X_{1}\right) \otimes \mathcal{M}_{x}^{\otimes 2 m_{1} n}\right) \neq 0
$$

holds for some $m_{1} \gg 1$.

To prove Lemma 3, we need the following lemma. 
Lemma 4. Let $M$ be a smooth projective $n$-fold and let $F$ be a nef and big line bundle on $M$. Then for every $q \geq 1$.

$$
\operatorname{dim} H^{q}\left(M, \hat{\mathcal{O}}_{M}(\nu F)\right) \leq O\left(\nu^{n-1}\right)
$$

holds as $\nu$ tends to infinity.

Proof of Lemma 4. By Kodaira's lemma ([8, Appendix]) there exists an effective divisor $E$ and a positive integer $\nu_{0}$ such that both $\nu_{0} F-E$ and $\nu_{0} F-E-$ $K_{X}$ is ample. Then by Kodaira's vanishing theorem, we have an isomorphism:

$$
H^{q}\left(M, \mathscr{O}_{M}(\nu F)\right) \simeq H^{q}\left(E, \mathscr{O}_{M}\left(\left.\nu F\right|_{E}\right)\right)
$$

for every $q \geq 1$ and $\nu>\nu_{0}$. Since

$$
\operatorname{dim} H^{q}\left(E, \mathscr{O}_{E}\left(\left.\nu F\right|_{E}\right)\right)=O\left(\nu^{n-1}\right),
$$

this completes the proof of Lemma 4 .

Q.E.D.

Proof of Lemma 3. Let $\mu: \hat{X}_{1} \rightarrow X_{1}$ be a resolution of singularity. By Lemma 4, we have

$$
H^{0}\left(\tilde{X}_{1}, \mathcal{O}_{\tilde{X} 1}\left(m_{1}(2 n+1) \mu^{*}\left(L \mid X_{1}\right) \otimes \mathcal{M}_{y}^{\otimes 2 m_{1} n}\right)\right) \neq 0
$$

holds for every $m_{1} \gg 1$ and $y \in \hat{X}_{1}$. If $X_{1}$ is normal then this completes the proof of Lemma 3. Suppose that $X_{1}$ is nonnormal. Let $D_{1}$ be the codimension 1 singular locus of $X_{1}$ and let $\bar{D}_{1}$ denote $\mu^{-1}\left(D_{1}\right)$. Then we have for every fixed positive integer $a$,

$$
\left.H^{0}\left(\tilde{X}_{1}, \mathscr{O}_{\tilde{X} 1}\left(\mu^{*}\left(m_{1}(2 n+1) L\right)-a \tilde{D}_{1}\right) \otimes M_{y}^{\otimes 2 m_{1} n}\right)\right) \neq 0
$$

for every $m_{1} \gg 1$ and $y \in \tilde{X}_{1}$. If we take a sufficiently large this completes the proof of Lemma 3.

Q.E.D.

Since $L$ is ample, by Serre's vanishing theorem, if we take $m_{1}$ sufficiently large

$$
H^{1}\left(X, \mathscr{O}_{X}\left(m_{1}(2 n+1) L\right) \otimes \mathfrak{O}_{X}\left(-X_{1}\right) \otimes M_{y}\right)=0
$$

for every $y \in X$, where $\mathscr{O}_{X}\left(-X_{1}\right)$ denotes the ideal sheaf of $X_{1}$. This implies that for every $y \in X-X_{1}$

$$
H^{0}\left(X, \mathscr{O}_{X}\left(m_{1}(2 n+1) L\right)\right) \rightarrow H^{0}\left(X_{1}, \mathscr{O}_{X_{1}}\left(m(2 n+1) L \mid X_{1}\right)\right) \oplus \mathscr{O}_{X} / \mathcal{M}_{y}
$$

is surjective if we take $m_{1}$ sufficiently large. Hence taking $m_{1}$ sufficiently large, if 
necessary, by Noetherian induction we may assume that the linear subsystem

$$
\left|\phi^{*} H^{0}\left(X_{1}, \mathscr{O}_{X_{1}}\left(m_{1}(2 n+1) L \mid X_{1} \otimes \mathcal{M}_{x}^{\otimes 2 m_{1} n}\right)\right)\right|
$$

of $\left|m_{1}(2 n+1) L\right|$ does not have base points on $X-X_{1}$. Let $\tau_{0}, \ldots, \tau_{M}$ be a basis of $\phi^{*} H^{0}\left(X_{1}, \mathscr{O}_{X_{1}}\left(m_{1}(2 n+1) L\right) \otimes M_{x}^{\otimes 2 m_{1} n}\right)$. We define a singular hermitian metric $H_{1 . x}$ by

$$
H_{1, x}=\frac{h^{\otimes m_{1}(2 n+1)}}{\sum_{j=0}^{M} h^{\otimes m_{1}(2 n+1)}\left(\tau_{j}, \tau_{j}\right)} .
$$

Then as before, curv $H_{1, x}$ is a closed current. Let $\varepsilon$ be a sufficiently small positive number. Let $\varphi_{1, t}$ be the function on $X$ defined by

$$
\varphi_{1, t}=\log \left(H_{x}^{\frac{\alpha-\varepsilon}{2 m}}\left(H_{1, x}^{\frac{t}{2 m}}\right) h^{-\left(n+\frac{1}{2}\right)(\alpha+t-\varepsilon)}\right)
$$

and let $\alpha_{1}$ be the positive number defined by

$$
\alpha_{1}=\sup \left(t \in \mathbf{R} \mid e^{-\varphi_{1, t}} \in L_{\mathrm{loc}}^{1}(X, x)\right\} .
$$

We set

$$
\mathscr{I}^{(1)}(t)=\mathscr{L}^{2}\left(\mathscr{O}_{X}, e^{-\varphi_{1, t}}\right) .
$$

Then by increasing $t$ we obtain a strictly decreasing sequence of ideals

$$
\mathfrak{O}_{X, x} \supset \mathscr{I}_{1, x}^{(1)} \supset \cdots .
$$

We set

$$
\mathscr{I}_{1}^{(1)}=\lim _{t \downarrow 0} \mathscr{I}^{(1)}\left(\alpha_{1}+t\right) .
$$

Then the stalk $\left(\mathscr{I}_{1}^{(1)}\right)_{x}$ of $\mathscr{I}_{1}^{(1)}$ at $x$ is $\mathscr{I}_{1, x}^{(1)}$ by the Noetherian property of coherent analytic sheaves. Let $X_{2}^{\prime}$ be the subscheme $V \oiint_{1}^{(1)}$ of $X$. Then by the construction $X_{2}^{\prime}$ is a subscheme of $X_{1}$. Let $X_{2}$ be a minimal dimensional irreducible component of $X_{2}^{\prime}$ containing $x$.

Lemma 5. We have the inequality:

$$
\alpha_{1} \leq n_{1} / n+O(\varepsilon) .
$$

To prove this lemma we need the following elementary lemma:

Lemma 6 . Let $b$ be a positive number. Then 


$$
\int_{0}^{1} \frac{r_{2}^{2 n_{1}-1}}{\left(r_{1}^{2}+r_{2}^{4 m_{1} n}\right)^{b}} d r_{2}=r_{1}^{\frac{n_{1}}{m_{1} n}-2 b} \int_{0}^{r_{1}^{-\frac{1}{2 m_{1} n}}} \frac{r_{3}^{2 n_{1}-1}}{\left(1+r_{3}^{4 m_{1} n}\right)^{b}} d r_{3}
$$

holds, where

$$
r_{3}=r_{2} / r_{1}^{1 / 2 m_{1} n}
$$

Suppose that $x$ is a regular point of $X_{1}$. Let $\left(z_{1}, \ldots, z_{n}\right)$ be a local coordinate on a neighbourhood $U$ of $x$ in $X$ such that

$$
U \cap X_{1}=\left\{p \in U \mid z_{n_{1}+1}(p)=\cdots=z_{n}(p)=0\right\} .
$$

We set $r_{1}=\left(\sum_{i=n_{1}+1}^{n}\left|z_{i}\right|^{2}\right)^{1 / 2}$ and $r_{2}=\left(\sum_{i=1}^{n_{1}}\left|z_{i}\right|^{2}\right)^{1 / 2}$. Then there exists a positive constant $C$ such that

$$
\sum_{j=0}^{M}\left|\tau_{j}\right|^{2} \leq C\left(r_{1}^{2}+r_{2}^{4 m_{1} n}\right)
$$

holds on a neighbourhood of $x$, where

$$
\left|\tau_{j}\right|^{2}=h\left(\tau_{j}, \tau_{j}\right)
$$

We note that there exists a positive integer $l$ such that

$$
\left.\sum_{i=0}^{N}\left|\sigma_{i}\right|^{2}\right)^{-1}=O\left(1 / r_{1}^{l}\right)
$$

on a neighbourhood of generic point of $X_{1} \cap U$. Then by Lemma 6 , we have the inequality $\alpha_{1} \leq n_{1} / n+O(\varepsilon)$.

For the next, suppose that $x$ is a singular point of $X_{1}$.

Let $\pi: \tilde{X} \rightarrow X$ be an embedded resolution of $X_{1}$ and let $X_{1}^{*}$ be the strict transform of $X_{1}$.

Lemma 7. Let $x_{1}$ be a point on $\pi^{-1}(x)$. Then there exist global sections

$$
\tau_{0}, \ldots \tau_{M} \in H^{0}\left(X, \mathscr{O}_{X}\left(m_{1}(2 n+1) L\right)\right)
$$

such that

$$
\left.\pi^{*}\left(\tau_{j}\right)\right|_{X_{1}^{*}} \in H^{0}\left(X_{1}^{*}, \mathscr{O}_{X_{1}^{*}}\left(\pi^{*}\left(m_{1}(2 n+1) L\right)\right) \otimes \mathcal{M}_{x_{1}}^{\otimes 2 m_{1} n}\right)
$$

holds for every $j$ and $\left\{\tau_{0}, \ldots, \tau_{M}\right\}$ is a basis for such sections.

The proof is the same as Lemma 3 . Let $x_{1}$ be a point on the strict transform $X_{1}^{*}$ such that $\pi\left(x_{1}\right)=x$. Let $\left(z_{1}^{1}, \ldots, z_{n}^{1}\right)$ be a local coordinate on a neighbourhood 
$\tilde{U}$ of $x_{1}$ such that

$$
\tilde{U} \cap X_{1}^{*}=\left\{p \in \tilde{U} \mid z_{n_{1}+1}^{1}(p)=\cdots z_{n}^{1}(p)=0\right\} .
$$

We define $\tilde{\tau}_{1}, \tilde{\tau}_{2}$ similarly as above. Then there exists a constant $C$ such that

$$
\pi^{*}\left(\sum_{j=0}^{M}\left|\tau_{j}\right|^{2}\right) \leq C\left(\tilde{r}_{1}^{2}+\tilde{r}_{2}^{4 m_{1} n}\right)
$$

holds. Then again by Lemma 6 and the uppersemicontinuity of the multiplicity, we have the inequality $\alpha_{1} \leq n_{1} / n+O(\varepsilon)$.

If $X_{2}=\{x\}$, then as before we have that

$$
H^{0}\left(X, \mathscr{O}_{X}\left(K_{X}+m L\right)\right) \rightarrow O_{X}\left(K_{X}+m L\right) / \mu_{x}
$$

is surjective for every

$$
m>\left(\alpha+\alpha_{1}\right)\left(n+\frac{1}{2}\right) .
$$

If $X_{2}$ is not $\{x\}$ we can continue the same process and obtain the strictly decreasing sequence of subvarieties

$$
X \supset X_{1} \supset X_{2} \supset \cdots .
$$

We see that there exists $k \leq n$ such that $X_{k}=\{x\}$. By Lemma 6 , we have that

$$
\sum_{i=0}^{k-1} \alpha_{\imath} \leq \frac{n(n+1)}{2 n}+\varepsilon
$$

holds, where $\alpha_{0}=\alpha$ and $\varepsilon$ is a positive number which we can take arbitrarily small. This implies that

$$
H^{0}\left(X, \mathscr{O}_{X}\left(K_{X}+m L\right)\right) \rightarrow O_{X}\left(K_{X}+m L\right) / \mathcal{M}_{x}
$$

is surjective for every

$$
m>\frac{n(n+1)}{2 n}\left(n+\frac{1}{2}\right)
$$

But we improve this estimate as

$$
m>\frac{n(n+1)}{2}
$$

by replacing $H^{0}\left(X, \mathscr{O}_{X}(m(2 n+1) L) \otimes \mathcal{M}_{x}^{\otimes 2 m n}\right)$ by $H^{0}\left(X, \mathscr{O}_{X}(m(N n+1) L) \otimes\right.$ $\mathcal{M}_{x}^{\otimes N m n}$ ) for a sufficiently large integer $N$ in Lemma 1 (with trivial change of con- 
stants in the argument after Lemma 1).

This completes the proof of Theorem 1 .

\section{A generalization}

The proof of Theorem 1 says a little bit more. We shall show the local version of Theorem 1 .

Definition 4. Let $X$ be a smooth projective variety and let $x$ be a point on $X$. Let $L$ be a nef and big line bundle on $X$. We set for $1 \leq d \leq \operatorname{dim} X$,

$\mu_{d}(L, x)=\inf \left\{\left(L^{d} V\right)^{1 / d} \mid V\right.$ is a $d$-dimensional subvariety of $X$ such that $\left.x \in V\right\}$.

Now we can state the local version of Theorem 1 .

Theorem 3. Let $X$ be a smooth projective variety defined over $\mathbf{C}$ of dimension $n$ and let $L$ be a nef and big line bundle on $X$. Let $x$ be an arbitrary point on $X$. Then $K_{X}+m L$ is generated by global sections at $x$ for every

$$
m>\sum_{d=1}^{n} \frac{d}{\mu_{d}(L, x)} .
$$

The proof is actually contained in the proof of Theorem 1 . Hence we omit it.

Remark 2. Let $X$ be a smooth projective $n$-fold and let $L$ be an ample line bundle on $X$. Then the proof of Theorem 1 implies that $K_{X}+m L$ gives a birational morphism for every $m>n(n+1)$. In fact $K_{X}+m L$ separates general two distinct point on $X$ for every $m>n(n+1)$ by a trivial modification of the proof of Theorem 1.

\section{REFERENCES}

[1] J. P. Demailly, A numerical criterion for very ample line bundles, J. Diff. Geom., 37 (1993), 323-374.

[2],$- L^{2}$ vanishing theorem for positive line bundles and adjunction theory, preprint (1994).

[3] L. Ein and R. Lazarsfeld, Global generation of pluricanonical and adjoint linear series on smooth projective threefolds, J. Amer. Math. Soc., 6 (1993), 875-903.

[4] T. Fujita, On polarized manifolds whose adjoint bundles are not semipositive, Algebraic Geometry, Sendai, 1985, Advanced Studies Pure Math., Vol. 10, North-Holland, Amsterdam (1987), 167-178. 
[5] - Remarks on Ein-Lazarsfeld criterion of spannedness of adjoint bundles of polarized threefold, preprint (1994).

[6] Y. Kawamata, The cone of curves of algebraic varieties, Ann. of Math., 119 (1984), 603-633.

[7] - Pluricanonical systems of minimal algebraic varieties, Invent. Math., 79, $567-588$ (1985).

[8] S. Kobayashi and T. Ochiai, Mappings into complex manifolds with negative first Chern class, J. Math. Soc. Japan, 23 (1971), 137-145.

[ 9 ] J. Kollár, Effective basepoint freeness, Math. Ann., 296 (1993), 595-605.

[10] S. Mori, Threefolds whose canonical bundles are not numerically effective, Ann. of Math., 116 (1982), 133-176.

[11] A. M. Nadel, Multiplier ideal sheaves and existence of Kähler-Einstein metrics of positive scalar curvature, Ann. of Math., 132 (1990), 549-596.

[12] I. Reider, Vector bundles of rank 2 and linear systems on algebraic surfaces, Ann. of Math., 127 (1988), 309-316.

[13] Y. T. Siu, An effective Matsusaka's big theorem, Ann. Inst. Fourier, 43 (1993), $1387-1405$.

[14] - Effective very ampleness, Invent. Math., 124 (1996), 563-571.

Department of Mathematics

Tokyo Institute of Technology

2-12-1 Ohokayama Megro

Tokyo, 152, Japan 